angiography had a worse prognosis ( $3 / 13$ died; $5 / 13$ delayed) than those with mural VOGM (2/10 had significant delay; none died). Of all cases in the series, $52 \%(61 \%$ of survivors) had no or minor delay. The features associated with the worst outcome were perinatal presentation, congestive heart failure, and choroidal angioarchitecture. (Fullerton HJ, Aminoff AR, Ferriero DM, Gupta N, Dowd CF. Neurodevelopmental outcome after endovascular treatment of vein of Galen malformations. Neurology November (2 of 2) 2003;61:1386-1390). (Reprints: Dr HJ Fullerton, Department of Neurology, University of California, San Francisco, Box 0114, San Francisco, CA 94143).

COMMENT. The majority of infants with vein of Galen malformation who are selected for endovascular treatment have a favorable neurodevelopmental outcome. Neonatal presentation, congestive heart failure, and choroidal as opposed to mural angioarchitecture are features carrying the worst prognosis. The overall mortality is $15 \%$. The majority of survivors have a good outcome with $52 \%$ having no delay and an additionai $9 \%$ having only mild delay in neurodevelopment.

\title{
INFANTILE NEUROPATHY WITH DIAPHRAGMATIC PALSY
}

'A group of 13 patients with early onset diaphragmatic palsy in association with a progressive neuropathy is presented from Great Ormond Street Hospital for Children, London, UK. Weakness and wasting developed over a period of weeks and showed a distal to proximal progression. The patients shared similar characteristics and diagnostic criteria that included early onset respiratory distress, low birth weight, slow motor nerve conduction velocities, and decrease in size of myelinated fibers on sural nerve biopsy. Mutations in 8 cases tested affected the same gene encoding immunoglobulin mu-binding protein 2 in patients with spinal muscular atrophy with respiratory distress type 1 (SMARD1). Histological examination of the spinal cord in one patient showed no evidence of SMA. Genetic and clinical heterogeneity is suggested. (Pitt M, Houlden H, Jacobs J, et al. Severe infantile neuropathy with diaphragmatic weakness and its relationship to SMARD1. Brain December 2003;126:2682-2692). (Respond: Dr Matthew Pitt, Department of Clinical Neurophysiology, Great Ormond Street Hospital for Children NHS Trust, Great Ormond Street, London WC1N 3JH, UK).

COMMENT. Infants and neonates presenting with severe respiratory distress and diaphragmatic palsy may have diaphragmatic SMA (SMARD1) or severe infantile axonal neuropathy with respiratory failure (SIANRF). The syndrome is clinically and genetically heterogeneous, some cases having mutations in the IGHMBP2 gene.

Clinical features of 29 infants with SMARD1 and 26 novel IGHMBP2 mutations are reported from Charite, Humboldt University, Berlin (Grohmann K et al. Ann Neurol 2003;54:719-724). Itrauterine growth retardation, weak cry, and foot deformities were the earliest manifestations. Patients presented at 1 to 6 months with respiratory distress due to diaphragmatic paralysis and progressive muscle weakness, predominantly distal lower limbs. Sensory and autonomic nerves are also affected. Diagnosis of SMARD1 is considered in infants with non-5q SMA, neuropathy, and muscle weakness and/or 
respiratory distress of unclear cause. Consanguineous parents of a child with SIDS should be checked for IGHMBP2 mutations.

NCS/EMG and muscle biopsy together are of diagnostic value in infants with arthrogryposis multiplex congenital (AMC), when the history, examination, and genetic evaluation are unrevealing. (Kang PB et al. Ann Neurol 2003;54:790-795). Analysis of 38 patients with AMC seen over a 23-year period at Children's Hospital, Boston, showed that the disorder was neurogenic in 8 , myopathic in 10 , other in 12 , and unknown in 8 . Neither EMG nor biopsy alone had consistently high sensitivities or specificities, but when concordant for neurogenic or myopathic findings, they were more accurate than either test alone.

\section{INFECTIOUS DISORDERS}

\section{HUMAN HERPESVIRUS-6 IN MESIAL TEMPORAL LOBE EPILEPSY}

Brain samples obtained from surgical resections in 8 patients with mesial temporal lobe epilepsy (MTLE) and 7 patients with neocortical epilepsy (NE) were quantitatively analyzed for HHV-6 in a study at the National Institute of Neurological Disorders and Stroke, Bethesda, MD. DNA obtained from 4 patients with MTLE had significantly elevated levels of HHV-6 determined by real-time PCR assay, but HHV-6 was not amplified in specimens from NE patients. Hippocampal sections from MTLE cases had the highest levels of HHV-6, subtype HHV-6B. Expression of HHV-6 was localized to astrocytes and confirmed by western blot analysis and immunohistochemistry. (Donati D, Akhyani N, Fogdell-Hahn A, et al. Detection of human herpesvirus- 6 in mesial temporal lobe epilepsy surgical brain resections. Neurology November (2 of 2) 2003;61:1405-1411). (Reprints: Dr S Jacobson, Neuroimmunology Branch, National Institute of Neurological Disorders and Stroke, NIH, Bethesda, MD 20892).

COMMENT. The detection of HHV-6 in hippocampal and temporal lobe astrocytes of patients with MTLE suggests a possible role for reactivation of the virus in infected astrocytes as a cause of MTLE. The specificity of the finding requires additional studies in patients without seizures. HHV-6 and roseola infantum are also associated with febrile convulsions, a proposed antecedent to mesial temporal sclerosis and MTLE. (see Progress in Pediatric Neurology III, PNB Publishers, 1997;pp24-28).

\section{RISK OF HEARING LOSS AFTER BACTERIAL MENINGITIS}

Presence of sensorineural hearing loss $(>25 \mathrm{~dB})$ was determined in 628 schoolaged children born between 1986 and 1994 and survived non-Hemophilus influenzae type B (HiB) bacterial meningitis between 1990 and 1995 in a study at VU Medical Center, Amsterdam; and University Medical Center and Wilhelmina Children's Hospital, Utrecht, the Netherlands. The incidence of hearing loss was 7\%; unilateral in $20(3 \%)$ and bilateral in $23(4 \%)$. Hearing loss was severe $(71-90 \mathrm{~dB})$ or profound $(>90 \mathrm{~dB})$, and 5 received 